\title{
Thermophoresis and Brownian Motion Effects on Magnetohydrodynamics Electro-Osmotic Jeffrey Nanofluid Peristaltic Flow in Asymmetric Rotating Microchannel
}

\author{
K. Venugopal Reddy ${ }^{1, *}$, M. Gnaneswara Reddy ${ }^{2}$, and O. D. Makinde ${ }^{3}$ \\ ${ }^{1}$ Department of Mathematics, Vignan's Lara Institute of Technology \& Science, Vadlamudi 522213, India \\ ${ }^{2}$ Department of Mathematics, Acharya Nagarjuna University Campus, Ongole 523001, India \\ ${ }^{3}$ Faculty of Military Science, Stellenbosch University, Private Bag X2, Saldanha 7395, South Africa
}

This article investigates with the thermophoresis and Brownian motion effects on MHD electro-osmotic Jeffrey nanofluid peristaltic flow in an asymmetric microchannel. Well established large wavelength and small Reynolds number approximations are invoked. Numerical solutions have been evaluated for the stream function, nanofluid velocity, nanofluid temperature and nanoparticle phenomena. The computed results for nanofluid velocity, temperature, and concentration fields are utilized to determine the skin-friction, Nusselt number, and Sherwood number. The graphical results have been presented and discussed for various involved parameters. The novel features of nanofluids made them potentially significant in heat and mass transfer mechanism occurring in medical and industrial processes like microelectronics, pharmaceutical processes, hybrid engines, thermal management of vehicles, refrigerator, chiller, gas temperature reduction and so forth. These processes bear tendency to enhance thermal conductivity and the convective heat transfer more efficiently than base fluid. This unique aspect made nanofluids the topic of interest in recent time via different fluid flow models. The problem at hand is one such application of nanofluids in peristaltic flow through the asymmetric rotating microchannel.

KEYWORDS: Peristaltic Flow, Electro-Osmotic Flow, Jeffrey Fluid, Heat Transfer, Mass Transfer, Nanofluid, Asymmetric Micro-Channel.

\section{INTRODUCTION}

Peristalsis is an instinctive movement of the longitudinal and circular muscles, mostly in the digestive tract but infrequently in other hollow tubes of the body, that occur in progressive wave contractions. This mechanism occurs in the esophagus, stomach, and intestines. The waves can be short, local reflexes or long, continuous contractions that travel the whole length of the organ, depending upon their location and what initiates their action. Since the seminal work of Latham, ${ }^{1}$ many investigations dealing with peristaltic flows under different flow geometries and assumptions have been presented by employing analytical, numerical and experimental approaches. Few recent studies in this direction may be mentioned. ${ }^{2-6}$

Nanofluid is a fluid containing nanometer-sized particles, called nanoparticles. These fluids are engineered

\footnotetext{
*Author to whom correspondence should be addressed.

Email: venugopal.reddy1982@gmail.com

Received: 11 April 2018

Accepted: 18 May 2018
}

colloidal suspensions of nanoparticles in a base fluid. The nanoparticles used in nanofluids are typically made of metals $(\mathrm{Al}, \mathrm{Cu})$, oxides $\left(\mathrm{Al}_{2} \mathrm{O}_{3}\right)$, carbides $(\mathrm{SiC})$, nitrides (AlN, SiN) or nonmetals (Graphite, carbon nanotubes) and the base fluid is usually a conductive fluid, such as water or ethylene glycol. Other base fluids are Oil and other lubricants, Bio-fluids and Polymer solutions. Nanoparticles range in diameter between 1 and $100 \mathrm{~nm}$. Nanofluids commonly contain up to a $5 \%$ volume fraction of nanoparticles to ensure effective heat transfer enhancements. The word "nanofluid" coined by $\mathrm{Choi}^{7}$ describes a liquid suspension containing ultra-fine particles (diameter less than $50 \mathrm{~nm}$ ). With the rapid advances in nanomanufacturing, many inexpensive combinations of liquid/particle are now available. Kuznetsov and $\mathrm{Nield}^{8}$ have studied the natural convective boundary-layer flow of a nanofluid past a vertical plate analytically. They used a model in which Brownian motion and thermophoresis effects were taken into account. Recently, Gnaneswara Reddy et al. ${ }^{9}$ have investigated the effects of the magnetic field and Ohmic heating on the viscous flow of a nanofluid towards 
a nonlinear permeable stretching sheet. Haddad et al. ${ }^{10}$ experimentally investigated natural convection in nanofluid by considering the role of thermophoresis and Brownian motion in heat transfer enhancement. Gnaneswara Reddy ${ }^{11}$ reported the influence of thermal radiation on natural convection boundary layer flow of a nanofluid past a vertical plate with uniform heat flux. The influence of magnetohydrodynamic and thermal radiation boundary layer flow of a nanofluid past a stretching sheet is studied by Gnaneswara Reddy. ${ }^{12}$ Moreover, many researchers have been investigated in the recent studies in Refs. [13-20].

By recent advancements in micro-fabrication, miniaturization takes into consideration for many applications spanning from biological to cooling of microelectronics applications. ${ }^{21-29}$ The operational state of many microinstruments deals with fluid flow in microscale channels (microchannel is the channel that its smallest dimension is between $10 \mu \mathrm{m}$ to $200 \mu \mathrm{m}$ ). Microfluidic devices offer many advantages such as a significant reduction in the consumption of required materials, ability to perform in-vitro experiments on the continuous flow in a manner similar to the real situation in a living biological system, being portable and vibration free. The heat transfer analysis on electro-osmotic flow in a slowly varying asymmetric micro-channel analyzed by Mondal and Shit. ${ }^{30}$ Saeid Hadian, et al. ${ }^{31}$ were studied the analytical study of the temperature distribution of the electro-osmotic flow in slit microchannels. The Micro-scale thermo-fluidic transport in two immiscible liquid layers subject to combined electroosmotic and pressure driven transport studied by Garai and Chakraborty. ${ }^{32}$ Shit et al. ${ }^{33}$ analyzed the two-layer electroosmotic flow and heat transfer in a hydrophobic microchannel with fluid-solid interfacial slip and zeta potential difference.

The current study is mainly motivated by the need to understand the MHD Electro-Osmotic Jeffrey nanofluid peristaltic flow in an asymmetric rotating microchannel. This model has important applications in heat transfer enhancement in the renewable energy systems and industrial thermal management, and also in material processing. The ND solve approach with Mathematica package is employed to study the effects of physical flow parameters such as magnetic parameter, osmosis parameter, Jeffrey fluid parameter, Brownian motion parameter and thermophoresis parameter and slip parameters on the fluid velocity component, temperature, and concentration distributions, and reduced skin-friction coefficient, Nusselt and Sherwood numbers and also trapping phenomenon has been investigated. The graphs are plotted and discussed for the variations of different involved parameters in details.

\section{MODEL FORMULATION}

The geometric model of the current study for the MHD electro-osmotic peristaltic flow of a nanofluid with different nanoparticles is depicted in Figure 1. We choose the

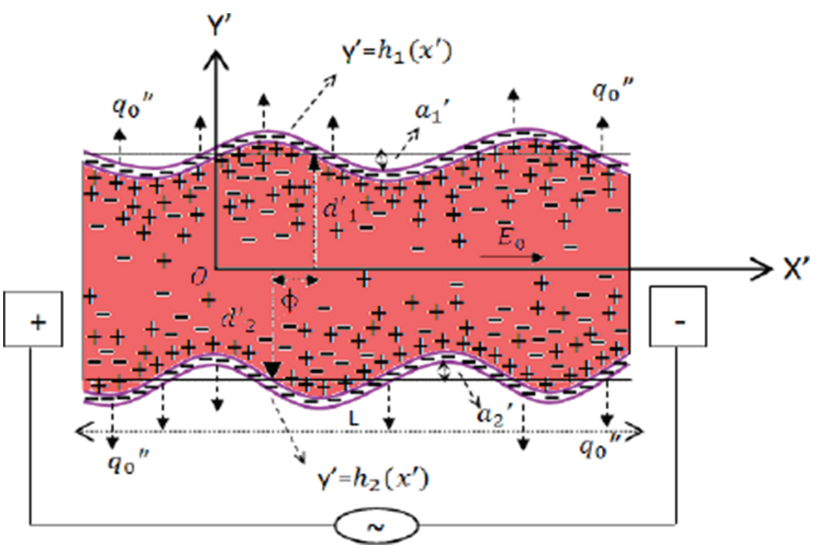

Fig. 1. Geometrical interpretation.

two-dimensional electro-osmotic peristaltic flow and heat transfer of viscous, incompressible fluid through a nonuniform micro-channel with negatively charged walls.

The flow is assumed to be asymmetric about $x^{\prime}$ and the liquid is flowing in the $x^{\prime}$-direction. The hydrophobic micro-channel is bounded by slowly varying walls at $y^{\prime}=h_{1}\left(x^{\prime}\right)$ and $y^{\prime}=h_{2}\left(x^{\prime}\right)$ respectively, in which the length of the channel $(L)$ is assumed to be much larger than the height, i.e., $L \gg\left(h_{1}+h_{2}\right)$. The geometrical expression of the wavy channels are given by

$$
\begin{aligned}
& h_{2}^{\prime}\left(x^{\prime}\right)=-d_{2}^{\prime}-a_{2}^{\prime} \cos \left(\frac{2 \pi x^{\prime}}{L}+\phi\right)
\end{aligned}
$$

\subsection{Electrical Potential Distribution}

The basic theory of electrostatics is related to the local net electric charge density $\rho_{e}$ in the diffuse layer of EDL and charge density is coupled with the potential distribution $\psi^{\prime}$ through the Poisson-Boltzmann equation for the symmetric electrolyte $^{27,28}$ given by

$$
\frac{d^{2} \psi^{\prime}\left(y^{\prime}\right)}{d y^{\prime 2}}=\frac{2 n_{0} e z_{v}}{\varepsilon} \sinh \left(\frac{e z_{v} \psi^{\prime}\left(y^{\prime}\right)}{k_{B} T_{a v}}\right)
$$

where $\eta_{o}$ represents the concentration of ions at the bulk, $\varepsilon$ is the charge of a proton, $z_{v}$ is the valence of ions, $e$ is the permittivity of the medium, $k_{B}$ is the Boltzmann constant and $T_{a v}$ is the boundary conditions for potential function are taken as

$$
\begin{array}{ll}
\psi^{\prime}\left(y^{\prime}\right)=\psi_{1}^{\prime} & \text { at } y^{\prime}=h_{1}^{\prime}\left(x^{\prime}\right), \\
\psi^{\prime}\left(y^{\prime}\right)=\psi_{2}^{\prime} & \text { at } y^{\prime}=h_{2}^{\prime}\left(x^{\prime}\right)
\end{array}
$$

where $\psi_{1}^{\prime}$ and $\psi_{2}^{\prime}$ are the electric potential at the upper and lower wall respectively.

Let us now introduce the following non-dimensional variables,

$\left[\psi_{O}, \psi_{1}, \psi_{2}\right]=\frac{e z_{v}}{k_{B} T_{a v}}\left[\psi^{\prime}, \psi_{1}^{\prime}, \psi_{2}^{\prime}\right], \quad y=\frac{y^{\prime}}{d_{1}^{\prime}} \quad$ and $\quad x=\frac{x^{\prime}}{L}$ 
The dimensionless form of Eqs. (1)-(2) and the PoissonBoltzmann equation defined in (3) take in the following form,

$$
\begin{gathered}
h_{1}(x)=\frac{h_{1}^{\prime}}{d_{1}^{\prime}}=1+a_{1} \cos (2 \pi x) \\
h_{2}(x)=\frac{h_{2}^{\prime}}{d_{1}^{\prime}}=-d-a_{2} \cos (2 \pi x+\phi)
\end{gathered}
$$

and

$$
\frac{d^{2} \psi_{O}}{d y^{2}}=k^{2} \sinh \left(\psi_{O}\right)
$$

where $a_{1}=a_{1}^{\prime} / d_{1}^{\prime}, a_{2}=a_{2}^{\prime} / d_{1}^{\prime}, d=d_{2}^{\prime} / d_{1}^{\prime}$ and $k=d_{1}^{\prime} / \lambda$ is defined as the electro-osmotic parameter, $\lambda$ is the reciprocal of the EDL thickness and is defined by $1 / \lambda=$ $\left(2 n_{o} e^{2} z_{v}^{2} / \varepsilon k_{B} T_{a v}\right)^{1 / 2}$. Thus the electro-osmotic parameter is inversely proportional to EDL thickness $\lambda$.

The dimensionless form of boundary conditions defined in (4) using the dimensionless variables (5) reduce to

$$
\begin{array}{ll}
\psi_{o}(y)=\psi_{1} & \text { at } y=h_{1}(x), \\
\psi_{o}(y)=\psi_{2} & \text { at } y=h_{2}(x)
\end{array}
$$

We assumed that the electric potential is much smaller than the thermal potential for which the Debye-Hiickel linearization principle can be approximated $\operatorname{as} \sinh (x) \approx x$. On the basis of this assumption, the solution of PoissonBoltzmann Eq. (8) takes in the form

$$
\frac{d^{2} \psi_{o}}{d y^{2}}=k^{2} \psi_{o}
$$

Finally, by employing the boundary conditions (9), the closed form solution of the Eq. (10) gives rise to

$$
\psi_{o}(y)=F_{1} \cosh (k y)+F_{2} \sinh (k y)
$$

\subsection{Nanofluid Model}

The governing equations for this investigation are obtained from the balance of continuity, temperature and concentration as

$$
\begin{gathered}
\frac{\partial u}{\partial x}+\frac{\partial v}{\partial y}=0 \\
\rho\left[\frac{\partial u}{\partial t}+u \frac{\partial u}{\partial x}+v \frac{\partial u}{\partial y}-2 \Omega v\right] \\
=-\frac{\partial p}{\partial x}+\frac{\partial}{\partial x}\left(S_{x x}\right)+\frac{\partial}{\partial y}\left(S_{x y}\right)+\rho g \beta\left(T-T_{0}\right) \\
+\rho g \beta^{*}\left(C-C_{0}\right)-\sigma B_{0}^{2} u+\rho_{e} E \\
\rho\left[\frac{\partial v}{\partial t}+u \frac{\partial v}{\partial x}+v \frac{\partial v}{\partial y}+2 \Omega u\right]=-\frac{\partial p}{\partial y}+\frac{\partial}{\partial x}\left(S_{y x}\right)+\frac{\partial}{\partial y}\left(S_{y y}\right)
\end{gathered}
$$

$$
\begin{gathered}
{\left[\frac{\partial T}{\partial t}+u \frac{\partial T}{\partial x}+v \frac{\partial T}{\partial y}\right]} \\
=\alpha \frac{\partial^{2} T}{\partial y^{2}}+\tau\left[D_{B}\left(\frac{\partial C}{\partial y} \frac{\partial T}{\partial y}\right)+\frac{D_{T}}{T_{0}}\left(\frac{\partial T}{\partial y}\right)^{2}\right] \\
+\frac{\mu}{\rho c_{p}\left(1+\lambda_{1}\right)}\left(\frac{\partial u}{\partial y}\right)^{2}+\frac{\sigma B_{0}^{2} u^{2}}{\rho c_{p}}+\frac{\sigma E^{2}}{\rho c_{p}} \\
{\left[\frac{\partial C}{\partial t}+u \frac{\partial C}{\partial x}+v \frac{\partial C}{\partial y}\right]=D_{m} \frac{\partial^{2} C}{\partial y^{2}}+\frac{D_{T}}{T_{0}} \frac{\partial^{2} T}{\partial y^{2}}}
\end{gathered}
$$

The constructive equations for an incompressible Jeffrey fluid are given by

$$
\begin{gathered}
\bar{T}=-\bar{p} \bar{I}+\bar{S} \\
\bar{S}=\frac{\mu}{1+\lambda_{1}}\left(\overline{\dot{\gamma}}+\lambda_{2} \overline{\bar{\gamma}}\right)
\end{gathered}
$$

where $\bar{p}$ is the pressure, $\bar{I}$ is the identity tensor, $\lambda_{1}$ is the ratio of relaxation to retardation times, $\lambda_{2}$ is the retardation time, $\dot{\gamma}$ is the shear rate and $\bar{S}$ is the extra stress tensor for Jeffrey fluid. In laboratory frame and dots over the quantities indicate differentiation with respect to time. The velocity slip conditions (Navier-Slip) at the fluid-solid interface are given by

$$
\begin{aligned}
& u+b_{1}\left(\frac{1}{1+\lambda_{1}}\right) \frac{d u}{d y}=0, \quad \text { at } y=h_{1}^{\prime}(x) \\
& u-b_{2}\left(\frac{1}{1+\lambda_{1}}\right) \frac{d u}{d y}=0, \quad \text { at } y=h_{2}^{\prime}(x)
\end{aligned}
$$

where $b_{1}$ and $b_{2}$ are the lengths of the channel walls $y=h_{1}^{\prime}$ and $y=h_{2}^{\prime}$ respectively.

Let us $U=u / U_{H S}$, with $U_{H S}=-\varepsilon E_{o} K T_{a \nu} / e z_{\nu} \mu$ the classical electro-osmotic flow velocity of microchannel in the absence of slip velocity, $k$ is the thermal conductivity, $u$ and $v$ are the velocity components in the $x$ and $y$-directions, respectively; $\sigma$ is the electric conductivity, $D_{B}$ is the Brownian motion coefficient, $D_{T}$ is the thermophoretic diffusion coefficient, $\tau$ is the ration of effective heat capacity of the nanoparticle material to heat capacity of the fluid, $T$ is the temperature, $T_{0}$ is the mean temperature and $C$ is the concentration. $\vec{\Omega}=\Omega \vec{k}, \vec{k}$ is the unit vector, $\vec{\Omega}=(0,0, \Omega)$ is the rotation vector, $\bar{R}$ is given by $\bar{R}^{2}=x^{2}+y^{2}, \bar{P}$ is the modified pressure. The equation of motion in the rotating frame has two additional terms i.e., $\rho \vec{\Omega} \wedge(\vec{\Omega} \wedge \vec{R})$ the centrifugal force and $2 \rho(\vec{\Omega} \wedge \vec{v})$ the Coriolis force. The flow is inherently unsteady in the laboratory frame $(x, y), \vec{R}=(u, v, 0)$. However, the flow becomes steady in a wave frame $(X, Y)$ moving away from the laboratory frame with speed $c$ in the direction of propagation of the wave. Taking $u$ and $v$ the velocity component in $x$ and $y$-directions, the transformation from the laboratory frame to the wave frame is given by

$$
\begin{gathered}
X=x-c t, \quad Y=y, \quad U=u-c, \\
V=v, \quad P(X)=p(x, t)
\end{gathered}
$$


where, $U$ and $V$ are the velocity components in the wave frame $(X, Y), P$ and $p$ are pressured in wave and fixed frame of references, respectively. The appropriate nondimensional variables for the flow defined as

$$
\begin{gathered}
\bar{x}=\frac{X}{\lambda_{1}}, \quad \bar{y}=\frac{Y}{d_{1}^{\prime}}, \quad \bar{u}=\frac{U}{c}, \quad \bar{v}=\frac{V}{c}, \quad \delta=\frac{d_{1}^{\prime}}{\lambda_{1}}, \\
\bar{p}=\frac{d_{1}^{\prime 2} P}{\mu c \lambda_{1}}, \quad \bar{t}=\frac{c t}{\lambda_{1}}, \quad h_{1}=\frac{h_{1}^{\prime}}{d_{1}^{\prime}}, \quad h_{2}=\frac{h_{2}^{\prime}}{d_{1}^{\prime}}, \\
\bar{S}=\frac{S d_{1}^{\prime}}{\mu c}, \quad d=\frac{d_{2}^{\prime}}{d_{1}^{\prime}}, \quad M=\sqrt{\frac{\sigma}{\mu}} B_{0} d_{1}^{\prime}, \quad a_{1}=\frac{a_{1}^{\prime}}{d_{1}^{\prime}}, \\
a_{2}=\frac{a_{2}^{\prime}}{d_{1}^{\prime}}, \quad d=\frac{d_{2}^{\prime}}{d_{1}^{\prime}} \quad R=\frac{\Omega_{1}}{\mu} a_{1}^{2}, \quad \operatorname{Re}=\frac{c d_{1}^{\prime}}{\nu}, \\
\theta=\frac{T-T_{0}}{T_{1}-T_{0}}, \quad E c=\frac{\rho \nu c_{p}}{c_{p}\left(T_{1}-T_{0}\right)}, \quad \operatorname{Pr}=\frac{K}{c^{2}}, \\
\beta_{1}=\frac{b_{1}}{d_{1}^{\prime}}, \quad \beta_{2}=\frac{b_{2}}{d_{1}^{\prime}}, \quad A=\frac{\sigma \bar{E}^{2} d_{1}^{2}}{\rho v c_{p}\left(T_{1}-T_{0}\right)}, \\
N t=\frac{\tau D_{T} D_{B}\left(T_{1}-T_{0}\right)}{T_{0} \nu}, \quad \Phi=\frac{C-C_{0}}{C_{1}-C_{0}}, \\
N b=\frac{\tau D_{B}\left(C_{1}-C_{0}\right)}{\nu \quad G r}, \quad G=\frac{\rho g \beta d_{1}^{\prime 2}\left(T-T_{0}\right)}{\mu c}, \\
G c=\frac{\rho g \beta^{*} d_{1}^{\prime 2}\left(C-C_{0}\right)}{\mu c}
\end{gathered}
$$

Introducing the dimensionless $\mid$ stream function $\downarrow \psi(x, y)$ such that

$$
u=\frac{\partial \psi}{\partial y}, \quad v=-\delta \frac{\partial \psi}{\partial x}
$$

By using (20)-(22) and under the assumptions of long wavelength $(\delta \ll 1)$ and low Reynolds number, the Eqs. (12)-(16) are reduced to

$\frac{1}{1+\lambda_{1}} \frac{\partial^{4} \psi}{\partial y^{4}}+\left(R-M^{2}\right) \frac{\partial^{2} \psi}{\partial y^{2}}+G r \frac{\partial \theta}{\partial y}+G c \frac{\partial \Phi}{\partial y}+\frac{\partial^{3} \psi_{0}}{\partial y^{3}}=0$

$$
\begin{gathered}
\frac{1}{\operatorname{Pr}} \frac{\partial^{2} \theta}{\partial y^{2}}+N b \frac{\partial \theta}{\partial y} \frac{\partial \Phi}{\partial y}+N t\left(\frac{\partial \theta}{\partial y}\right)^{2}+\frac{E c}{\left(1+\lambda_{1}\right)}\left(\frac{\partial^{2} \psi}{\partial y^{2}}\right)^{2} \\
+M^{2}\left(\frac{\partial \psi}{\partial y}\right)^{2}+A=0 \\
\frac{\partial^{2} \Phi}{\partial y^{2}}+\frac{N t}{N b} \frac{\partial^{2} \theta}{\partial y^{2}}=0
\end{gathered}
$$

The corresponding boundary conditions for the stream function and temperature in the wave frame are given by

$$
\begin{gathered}
\psi=\frac{q}{2}, \quad \frac{\partial \psi}{\partial y}+\beta_{1}\left(\frac{1}{1+\lambda_{1}}\right) \frac{\partial^{2} \psi}{\partial y^{2}}=0, \frac{\partial^{2} \psi}{\partial y^{2}}=-1 \\
\text { at } h_{1}(x)=1+a \cos (x) \\
\psi=-\frac{q}{2}, \frac{\partial \psi}{\partial y}-\beta_{2}\left(\frac{1}{1+\lambda_{1}}\right) \frac{\partial^{2} \psi}{\partial y^{2}}=0, \frac{\partial^{2} \psi}{\partial y^{2}}=-1 \\
\text { at } h_{2}(x)=-d-b \cos (2 \pi x+\varphi)
\end{gathered}
$$

$$
\begin{gathered}
\theta=0 \quad \text { at } h_{1}(x)=1+a \cos (x) \\
\theta=1 \quad \text { at } h_{2}(x)=-d-b \cos (2 \pi x+\varphi) \\
\phi=0 \quad \text { at } h_{1}(x)=1+a \cos (x) \\
\phi=1 \quad \text { at } h_{2}(x)=-d-b \cos (2 \pi x+\varphi)
\end{gathered}
$$

where $M$ is the Hartmann number, $P r$ is the Prandtl number, $E c$ is the Eckert number, $\lambda_{1}$ is the Jeffrey fluid parameter, $R$ is the rotation parameter, $\phi$ is phase difference, $\beta_{1}$ and $\beta_{2}$ are the slip parameters, $A$ is the Joule heating parameter, $G r$ is the thermal Grashof number, $G c$ is the solutal Grashof number and $q$ is the flux in the wave frame.

The dimensionless skin-friction coefficient, Nusselt number (heat transfer coefficient) and Sherwood number are given by

$$
\begin{gathered}
C_{f}=\left.\left(\frac{1}{1+\lambda_{1}}\right) \frac{\partial u}{\partial y}\right|_{y=h_{1}, h_{2}}, \quad N u=-\left.\frac{\partial \theta}{\partial y}\right|_{y=h_{1}, h_{2}}, \\
S h=-\left.\frac{\partial \Phi}{\partial y}\right|_{y=h_{1}, h_{2}},
\end{gathered}
$$
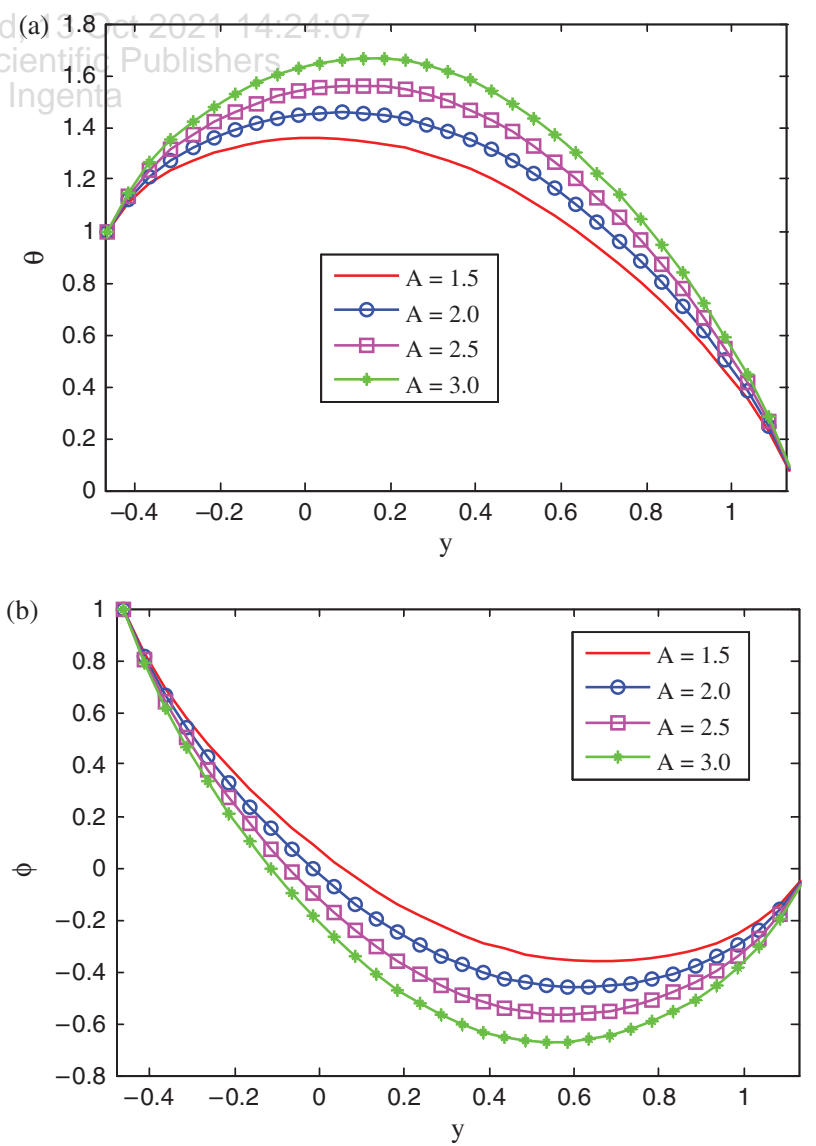

Fig. 2. (a) Effect of $A$ on temperature. (b) Effect of $A$ on nanoparticle concentration. 


\section{NUMERICAL SOLUTION}

The governing Eqs. (23)-(25) of an electro-osmotic Jeffrey nanofluid model are coupled and highly nonlinear. Getting the analytic solution is impossible. These equations along with the associated boundary conditions (26)-(31) are solving numerically by adopting NDSolve in Mathematica using Shooting method.

\section{RESULTS AND DISCUSSION}

As a result of the numerical calculations, the dimensionless stream function, velocity, temperature, and concentration
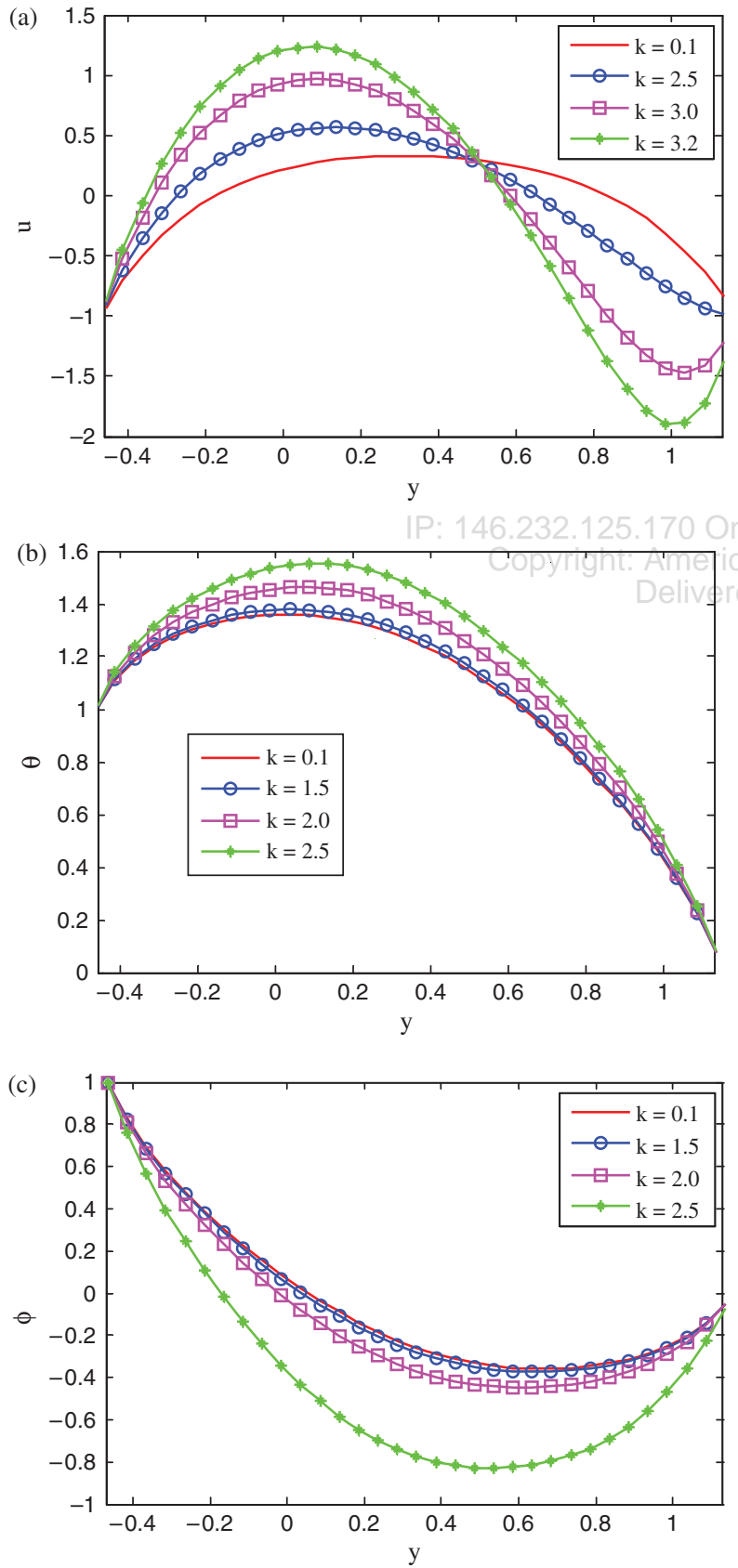

Fig. 3. (a) Effect of $k$ on velocity. (b) Effect of $k$ on temperature (c) Effect of $k$ on nanoparticle concentration.

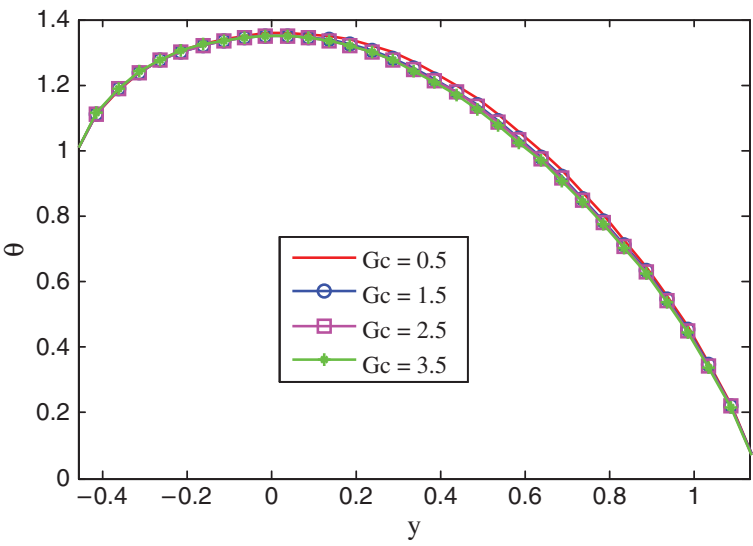

Fig. 4. Effect of $G c$ on temperature.

distributions for the flow under the impact of emerging fluid dynamical parameters considered through plots. Also, the numerical values of the skin-friction coefficient, Nusselt number, and Sherwood number are presented in the table. In the present study, the following default parametric values are adopted. The trapping phenomenon of the streamlines is also analyzed.

$$
\begin{gathered}
a=0.5, \quad b=0.8, \quad \phi=\frac{\pi}{3}, \quad d=1.0, \quad R=3.5, \\
d, 13=3, \quad Q=2.0, \beta_{1}=0.03, \quad \beta_{2}=0.02, \\
\text { cienget } \psi_{1}=0.3, \quad \psi_{2}=0.3, \quad k=0.1, \quad \operatorname{Pr}=0.7, \\
E c=1, \quad \lambda_{1}=1.5, \quad G c=0.5, \quad G r=0.5, \quad B r=1, \\
N b=0.1, \quad N t=0.1, \quad A=2
\end{gathered}
$$

Figure 2(a) depicts that the temperature enhances significantly with a rise in the volumetric heat generation/absorption (Joule heating) parameter $A$ near the central part of the channel, whereas it diminishes near the channel wall. Figure 2(b) illustrate the effect of the Joule heating parameter $A$ respectively on the concentration profile. It is noticed from the figures that the concentration profiles decrease with an increase in the values of $A$.

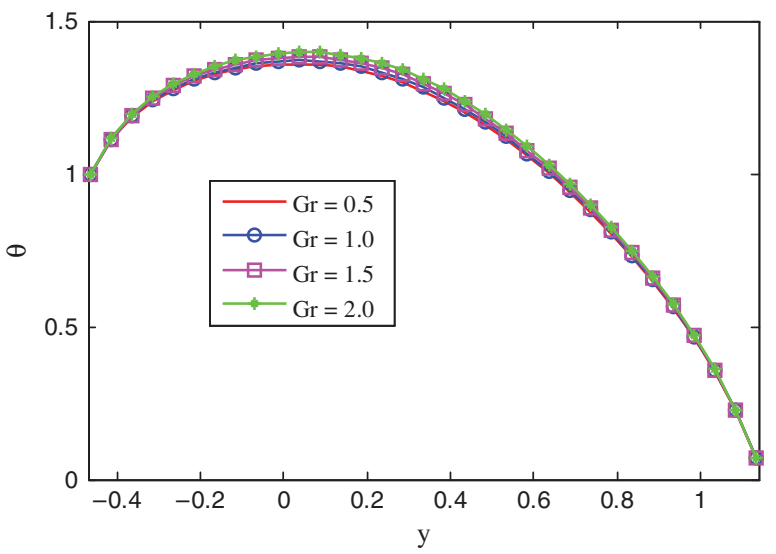

Fig. 5. Effect of $G r$ on temperature. 
Figure 2(a) depicts that the temperature enhances significantly with a rise in the volumetric heat generation/ absorption (Joule heating) parameter near the central part of the channel, whereas it decreases near the channel wall. It is worthwhile to mention here that the Joule heating parameters $A>0$ represent for volumetric heat generation meaning heat is externally added to the fluid through the channel walls. This enables us to observe higher temperature at the central region of the channel. Similarly for Joule heating parameter $A<0$ indicates for volumetric heat absorption in which the heat is lost through the wall
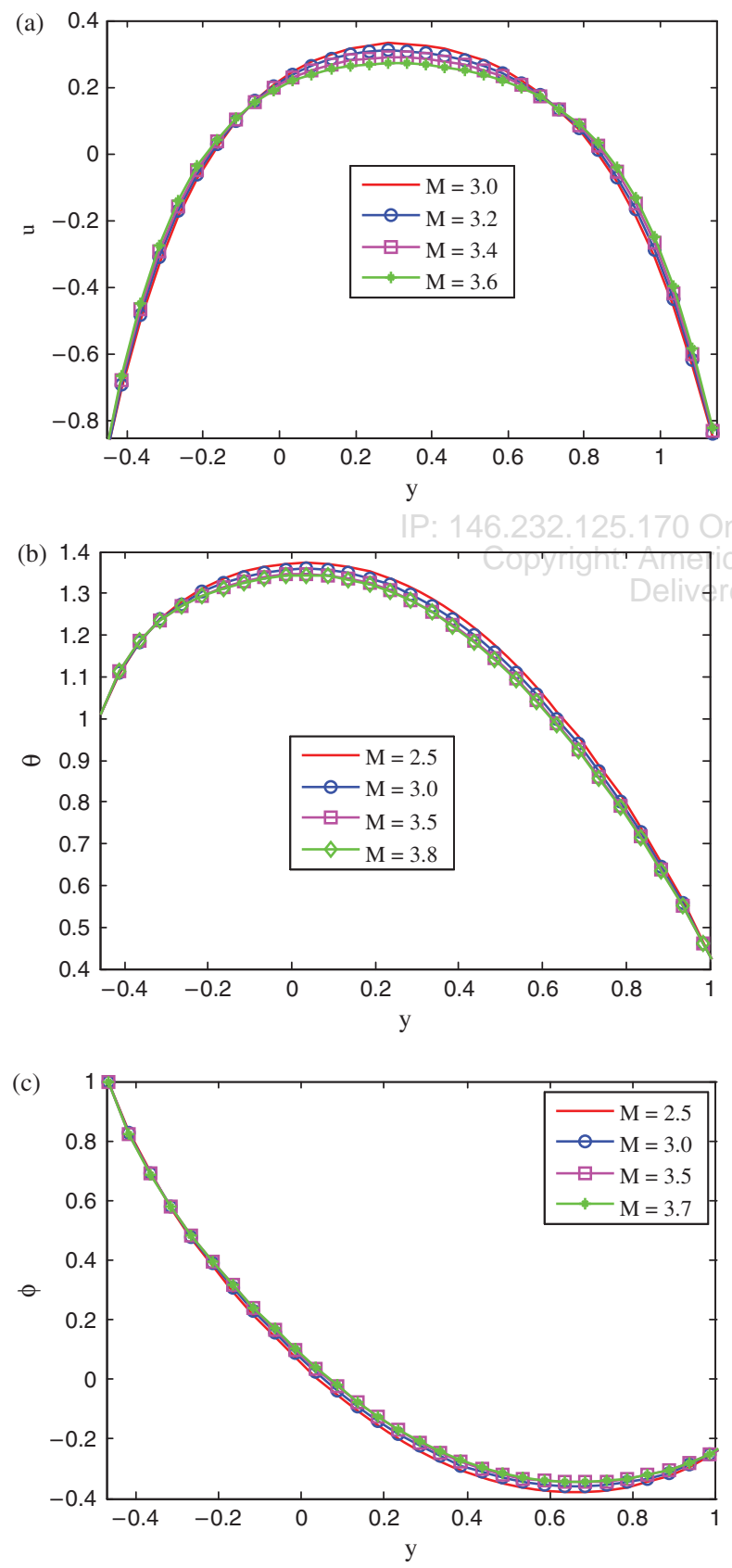

Fig. 6. (a) Effect of $M$ on velocity. (b) Effect of $M$ on temperature. (c) Effect of $M$ on nanoparticle concentration. of the channel and makes cooling of the system. In the absence of Joule heating parameter $A=0$, the temperature distribution is independent of any external heating/cooling and is very similar to the classical heat transfer phenomena. This figure also emphasizes that as Joule heating parameter rises, the temperature of the fluid located near the channel walls is reduced. This is because of the existence of temperature jump factor and the energy is transported by convection.

Figure 3(a) analyzes that the axial velocity rises with an enhance in the electro-osmotic parameter $k$. Since the electro-osmotic parameter $k$ is the reciprocal of EDL, then as $k$ increases, the thickness of EDL decreases thereby bulk fluid motion enhances. The electro-osmotic velocity $u$ is maximum for any value of $k$ in the central region of the microchannel. It indicates that an increase in the height of the microchannel, as well as a decrease in the Debye thickness of the microchannel, causes a rise in axial velocity. This may be explained by the fact that for thick EDLs, the effect of large zeta potential at the shear plane implicitly ensures a large extent to transport of mobile ions in the EDL of the microchannel. The influence of electro-osmotic parameter $k$ on the temperature profile is shown in Figure 3(b). It is evident that an increase in the
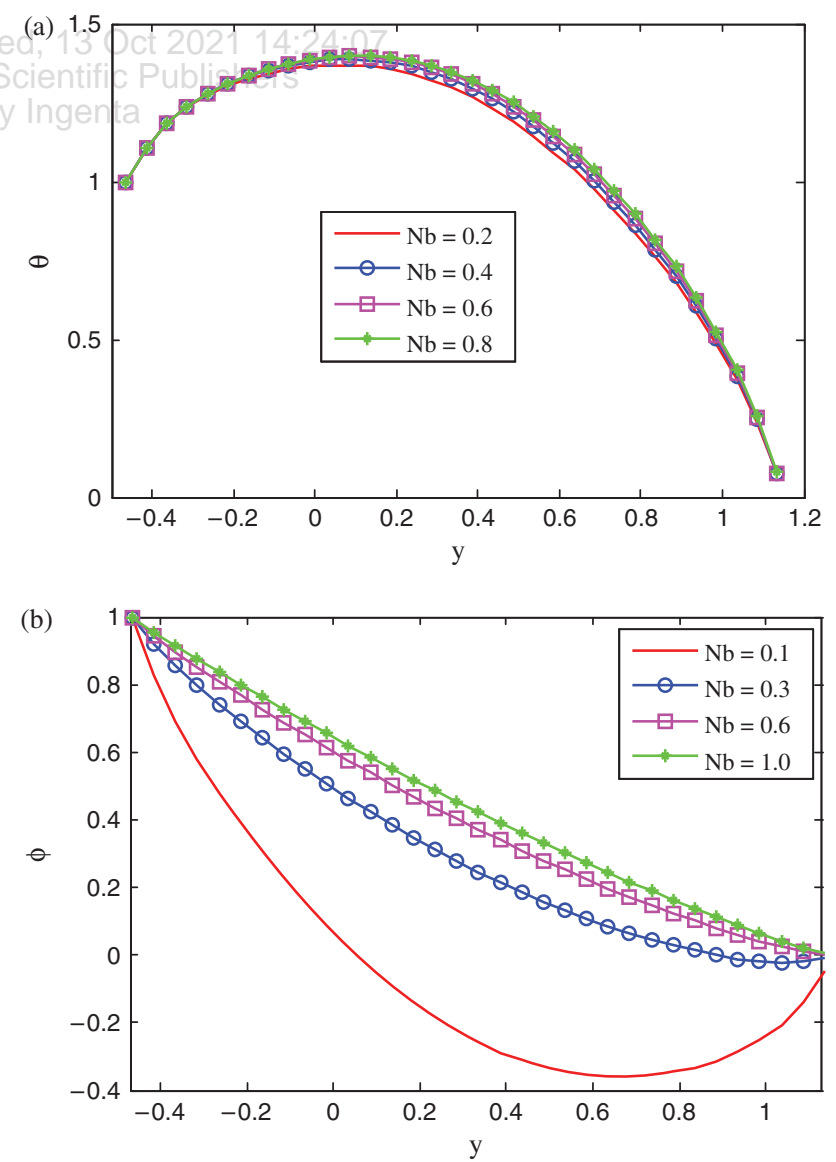

Fig. 7. (a) Effect of $N b$ on temperature. (b) Effect of $N b$ on nanoparticle concentration. 
electro-osmotic parameter $k$ enhances the temperature profiles of the flow. Figure 3(c) illustrate the effects of the electro-osmotic parameter $k$ on the concentration profiles. It is noticed from the figures that the concentration profiles decrease with an increase in the values of $k$.

Figures 4-5 illustrate that the influence of both thermal Grashof number $\mathrm{Gr}$ and solutal Grashof number $G c$ on temperature distribution. It observes that the temperature rises with the higher values of $G r$, whereas the temperature depresses with an enhancing the values of $G c$. We can see that fluid velocity increases with increase in $G r$. Grashof number is the ratio of buoyancy and viscous force. An increase in $G r$ enhances temperature gradient which increases buoyancy forces and fluid flow rises.

The velocity profiles for different values of the magnetic parameter $M$ are depicted in Figure 6(a). The reduction in the profiles is due to an induced in the magnetic field in an electrically conducting fluid that stimulates a drag force known as a Lorentz force which resists the fluid motion as shown in the Figure 6(a). As a result of the opposition to the fluid motion due to Lorentz force, an additional extra work is done that changes the thermal energy. The effect of the Hartmann number $M$ on the temperature profile is displayed in Figure 6(b). It is clear from these plots
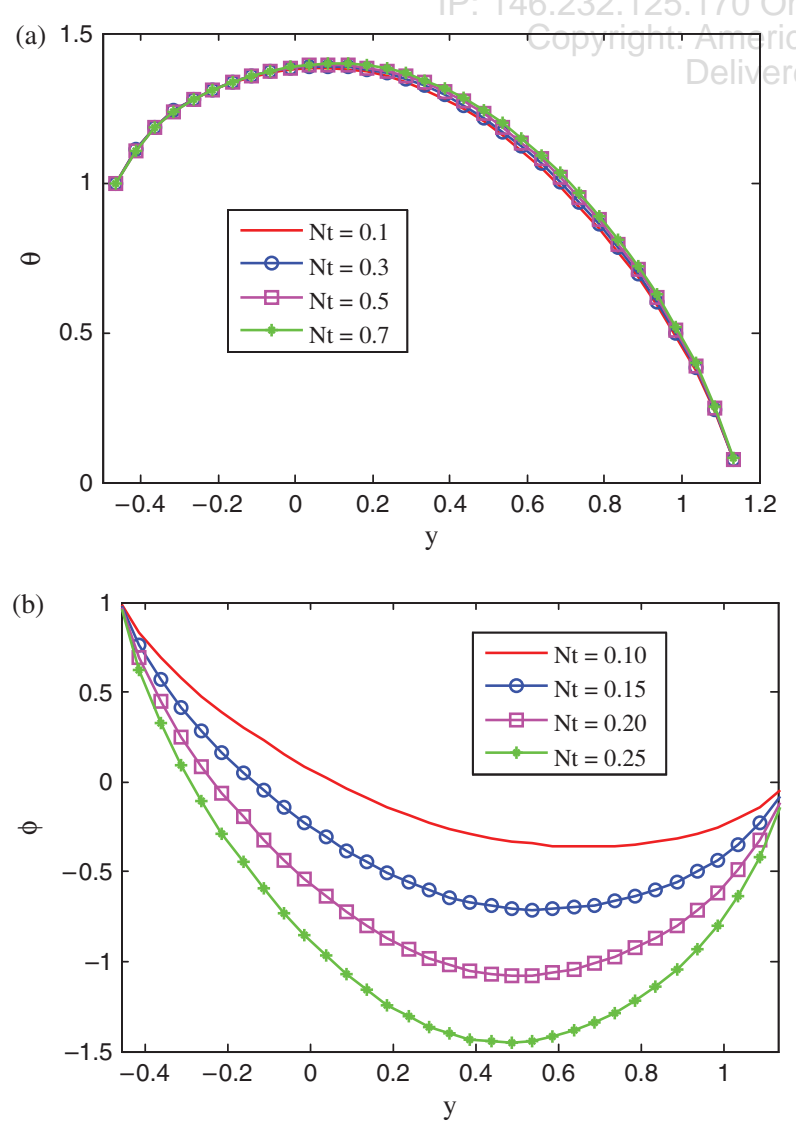

Fig. 8. (a) Effect of $N t$ on temperature. (b) Effect of $N t$ on nanoparticle concentration. that the energy reduces with an increase in the Hartmann number. Magnetic field takes the role of a retarding force. This force slows down the motion of the fluid particles. As a result of this, the nanoparticle concentration diminishes by enhancing Hartmann number $M$ which is evident from Figure 6(c).

The influence of Brownian motion parameter on the temperature is shown Figure 7(a). There is a substantial increase in the temperature with an increase in $\mathrm{Nb}$. As the Brownian motion effect strengthens this corresponds to the effective movement of nanoparticles from the wall to the fluid which results in the significant increase in the temperature distribution. Here also the same effect as a Brownian parameter on the temperature field. Figure 7(b) illustrates the nanoparticle concentration profiles for various values of $N b$. It is noticed that the concentration profile increases with an increase in $N b$. It is interesting to see that the smaller values of $\mathrm{Nb}$ strongly affect the concentration function.

Figure 8(a) shows that the temperature distribution of fluid for different values of thermophoresis parameter $\mathrm{Nt}$. It is seen from Figure 8(a), the temperature distribution increases with increasing $N t$. It is clear from Figure 8(b) that the magnitude of concentration function is significant decreases with an increase in $N t$. From the physical point of view gradual increase in the strength of
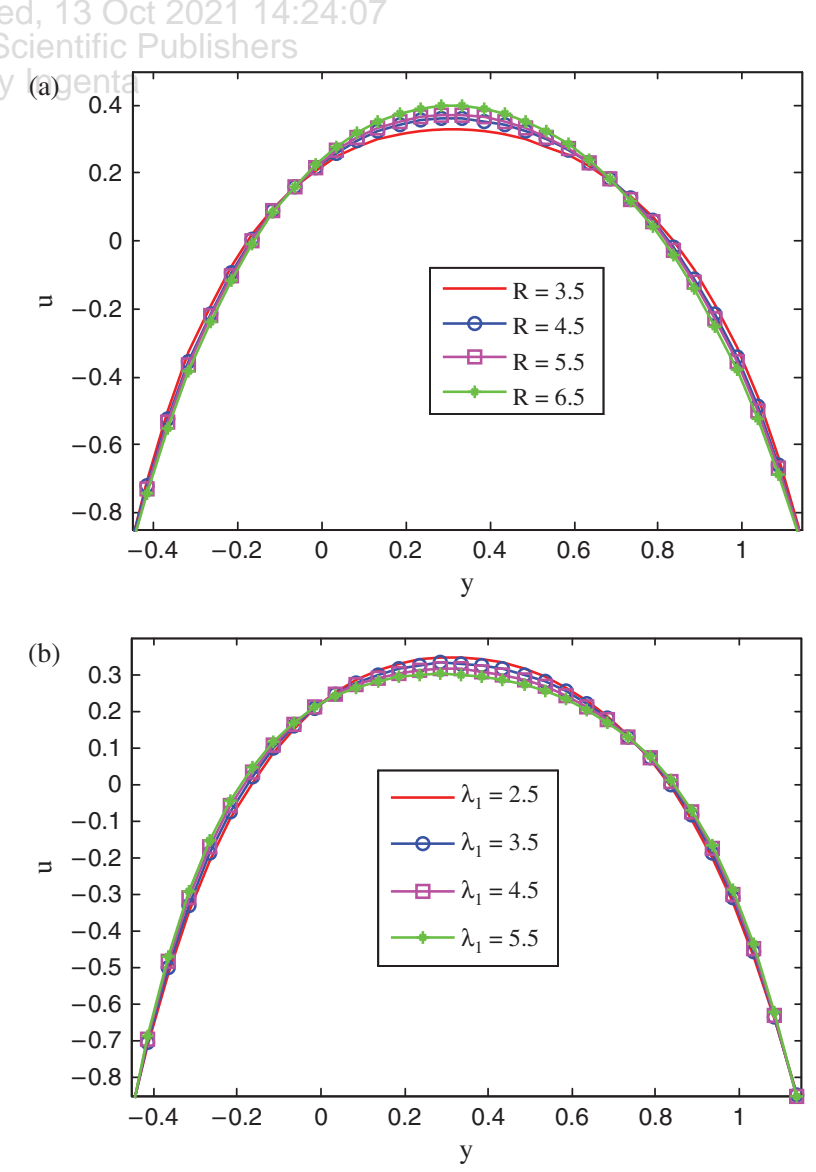

Fig. 9. (a) Effect of $R$ on velocity. (b) Effect of $\lambda_{1}$ on velocity. 
thermophoretic effect results in the larger mass flux due to temperature gradient which decreases the nanoparticles concentration.

Figure 9(a) depicts that the effect of rotation parameter $R$ is shown on velocity profile. It illustrates that the velocity distribution rises with an enhance in $R$. Figure 9(b) portrays the response of Jeffrey fluid parameter $\lambda_{1}$ on the velocity distribution. From the figure, it is seen that an increase in the Jeffrey fluid parameter reduces the fluid flow rate.

The phenomenon of trapping is another interesting topic in peristaltic transport. The formation of an internally circulating bolus of the fluid by closed streamlines is called trapping and this trapped bolus pushed ahead along the peristaltic wave. The effects of Hartmann number $(M)$ and Jeffrey fluid parameter $\left(\lambda_{1}\right)$ are analyzed on streamlines from Figures 10-11. The size of trapped bolus rises with increasing in $M$ from Figures 10(a)-(b). The size of trapped bolus diminishes as the higher values of $\lambda_{1}$ in Figures 11(a)-(b).

The numerical results for Brownian motion parameter on temperature and nanoparticle concentration are presented in Figure 12 in the absence Hartmann number $M=0$ and $k=0$. From these figures that our results are

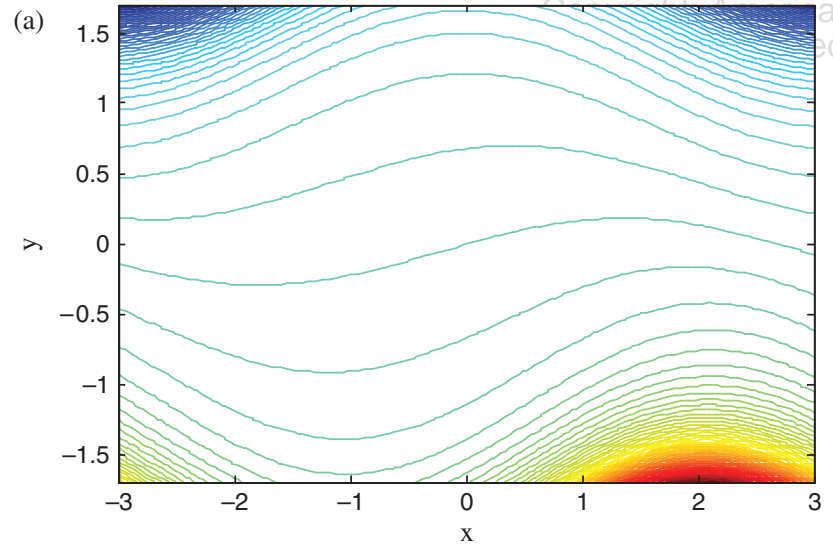

(b)

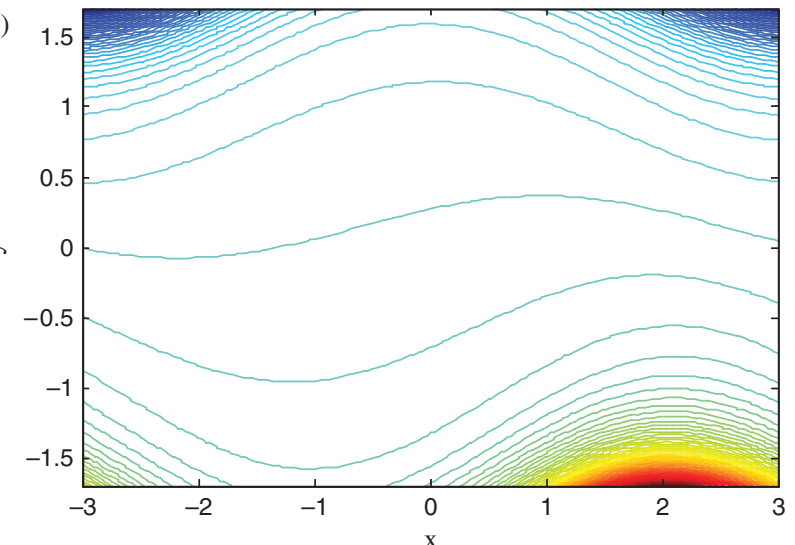

Fig. 10. (a) Streamlines for $M=0.5$. (b) Streamlines for $M=2.0$.

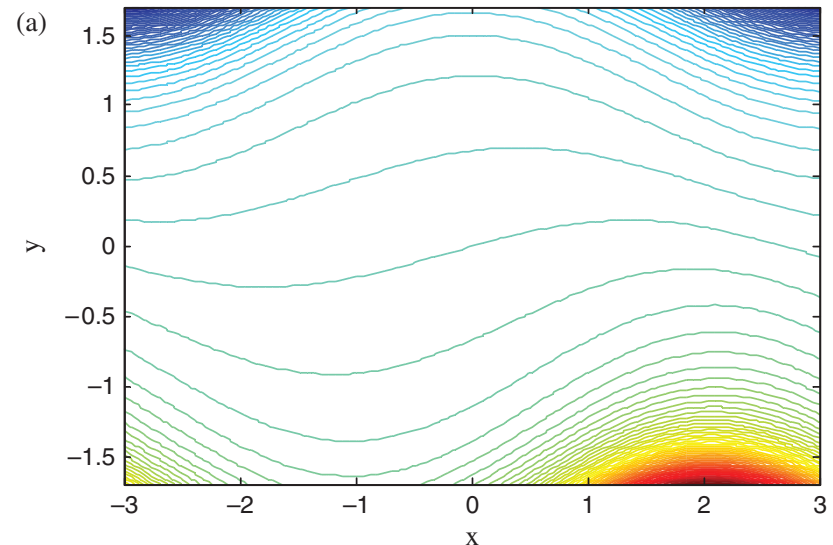

(b)

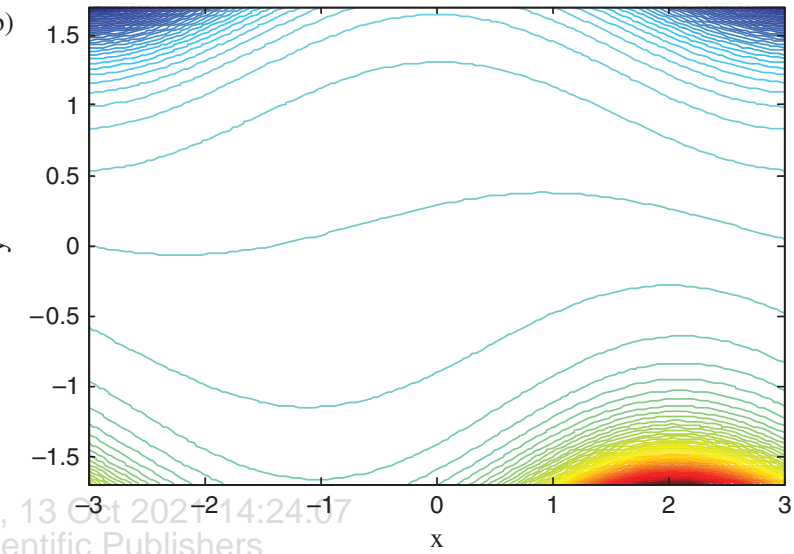

Fig. 11. (a) Streamlines for $\lambda_{1}=0.5$. (b) Streamlines for $\lambda_{1}=2.0$.

in good agreement with the earlier published work by Gnaneswara Reddy and Makinde. ${ }^{19}$

The influence of various emerging pertinent parameters to see the behavior on the skin-friction coefficient, Nusselt number and Sherwood number at the upper wall $h_{1}$ is shown in Table I. It is observed that both skinfriction coefficient and Sherwood number enhances with an increase in Hartman number while the reverse trend to the Nusselt number. It can be found that skin-friction

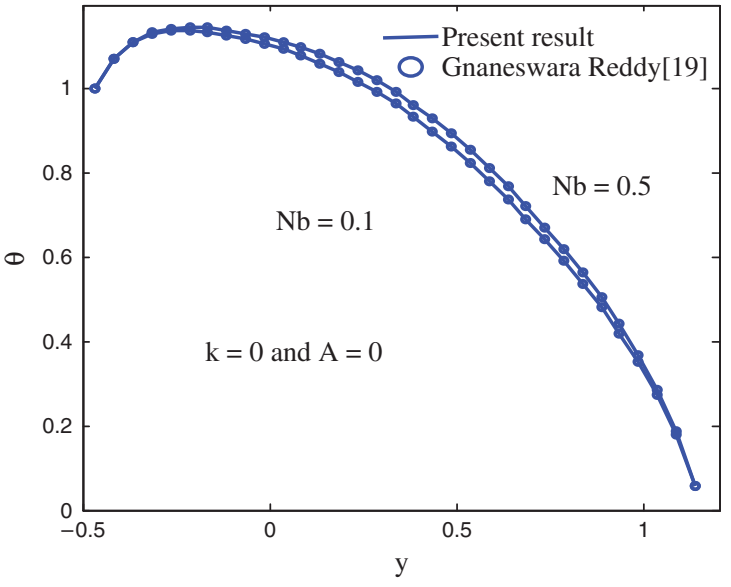

Fig. 12. Comparision on nanoparticle concentration. 
Table I. Numerical values of the skin-friction, Nusselt number and Sherwood number at the upper wall $h_{1}$ when $x=0.3$.

\begin{tabular}{|c|c|c|c|c|c|c|c|c|c|c|}
\hline$M$ & $\lambda_{1}$ & $G r$ & $G c$ & $N t$ & $\mathrm{Nb}$ & $k$ & $A$ & $C_{f}$ & $\mathrm{Nu}$ & $S h$ \\
\hline 2.9 & 1.5 & 0.5 & 0.5 & 0.1 & 0.1 & 0.1 & 1.5 & 0.0369724 & 1.36111 & -0.499375 \\
\hline 3 & & & & & & & & 0.0382421 & 1.35449 & -0.492746 \\
\hline \multirow[t]{15}{*}{3.1} & & & & & & & & 0.0392645 & 1.34854 & -0.486799 \\
\hline & 1.7 & & & & & & & 0.0352532 & 1.33816 & -0.476416 \\
\hline & 1.9 & & & & & & & 0.0326483 & 1.32363 & -0.461892 \\
\hline & & 0.6 & & & & & & -0.033508 & 2.2427 & -1.98236 \\
\hline & & 0.7 & & & & & & -0.036512 & 1.76887 & -1.71017 \\
\hline & & & 0.6 & & & & & -0.028121 & 1.91784 & -1.53677 \\
\hline & & & 0.7 & & & & & -0.007723 & 1.7343 & -1.72611 \\
\hline & & & & 0.2 & & & & 0.0137336 & 1.86761 & -3.58805 \\
\hline & & & & 0.3 & & & & 0.0473416 & 2.12779 & -5.81128 \\
\hline & & & & & 0.2 & & & -0.043237 & 1.63245 & -0.731283 \\
\hline & & & & & 0.3 & & & -0.061614 & 1.39803 & -0.247151 \\
\hline & & & & & & 0.2 & & 0.0382413 & 1.35448 & -0.492735 \\
\hline & & & & & & 0.3 & & 0.0382351 & 1.35444 & -0.492703 \\
\hline & & & & & & & 1.6 & 0.0382421 & 1.39638 & -0.534636 \\
\hline & & & & & & & 2.0 & 0.0382421 & 1.56394 & -0.702199 \\
\hline
\end{tabular}

coefficient and the Nusselt number decreases with an increase in the material parameter $\lambda_{1}$ while the opposite behavior to the Sherwood number. It can be analyzed that skin-friction coefficient and Nusselt number depresses with an increase in the thermal Grashof number and concentration Grashof number while it enhances to the Sherwood number. It is depicted that the skin-friction coefficient with an enhancing in the thermophoresis parameter $N t$ while a reverse trend observed to the and Nusselt number and Sherwood number whereas a quite opposite behavior observed to the Brownian motion parameter $\mathrm{Nb}$. It can be depicted that the skin-friction coefficient and Sherwood number enhances with an increase in the electro-osmosis parameter $k$ while opposite trend observed to the Nusselt number. It can be found that the skin-friction coefficient and Nusselt number enhances with an increase in the Jeffrey fluid parameter $A$ while the quite reverse nature is observed to the Sherwood number.

\section{CONCLUSIONS}

The influences of the thermophoresis and Brownian motion effects on MHD electro-osmotic Jeffrey nanofluid peristaltic flow in an asymmetric microchannel have been studied. The formulated equations for the flow are nondimensionalised and simplified using longwave length and low Reynolds number approximations. The nondimensional equations of the flow are solved numerically. Calculated results are represented graphically to show the importance of some parameters on the flow. It is observed that:

- Electro-osmotic motion signifies strong dependent on Debye length.

- A rise in thermal Grashof parameter needs to be guided because it contributes significantly to the increase of the temperature profile while the concentration Grashof parameter resists the free flow of temperature fluid profile. - The temperature profile enhances with an increase in thermophoresis parameter and Brownian motion parameter.

- Nanoparticle volume fraction profile depresses with an increase in Brownian motion parameter.

- Enhance in Hartmann number diminishes the bolus of the trapping phenomenon whereas reverse nature observed to the Jeffrey fluid parameter.

- The skin-friction coefficient and Sherwood number rises with an increase in Hartman number while the reverse trend to the Nusselt number.

\section{References and Notes}

1. T. W. Latham, Fluid Motion in a Peristaltic Pump, MIT, Cambridge, MA (1966).

2. A. H. Shapiro, M. Y. Jaffrin, and S. L. Weinberg, J. Fluid Mech. 37, 799 (1969).

3. M. Gnaneswara Reddy and K. Venugopal Reddy, J. Nige. Math. Soc. 35, 227 (2016).

4. M. Gnaneswara Reddy, K. Venugopal Reddy, and O. D. Makinde, Alex. Eng. Journal 55, 1841 (2016).

5. M. Gnaneswara Reddy, K. Venugopal Reddy, and O. D. Makinde, International Journal of Applied and Computational Mathematics 3, 3201 (2017).

6. A. Garai and S. Chakraborty, Int. J. Heat and Mass Tran. 52, 2660 (2009).

7. S. U. S. Choi, Enhancing thermal conductivity of fluids with nanoparticles, The Proceedings of the 1995 ASME Int. Mechanical Engineering Congress and Exposition, ASME, San Francisco, USA (1995), FED 231/MD 66, pp. 99-105.

8. A. V. Kuznetsov and D. A. Nield, Int. J. Therm. Sci. 49, 243 (2010).

9. M. Gnaneswara Reddy, P. Padma, and B. Shankar, J. Nanofluids 5, 459 (2016).

10. Z. Haddad, A. Nada, F. Oztop, and A. Mataoui, Int. J. Therm Sci. 57, 152 (2012).

11. M. Gnaneswara reddy, Int. J. Heat and Technology 32, 1 (2014).

12. M. Gnaneswara Reddy, J. Sci. Res. 6, 257 (2014).

13. M. Elshahed and M. H. Haroun, Math. Prob. Eng. 6, 663 (2005).

14. E. F. Elshehawey, N. T. Eldabe, E. M. Elghazy, and A. Ebaid, App. Math. Comp. 182, 140 (2006).

15. T. Hayat and N. Ali, Physica A 370, 225 (2006).

16. M. H. Haroun, Commun. Non-Linear Sci. Numer. Simul. 12, 1464 (2007).

17. J. Buongiorno, D. C. Venerus, N. Prabhat, T. McKrell, J. Townsend, R. Christianson, Y. V. Tolmachev, P. Keblinski, L. W. Hu, J. L. Alvarado, and I. C. Bang, J. Appl. Phys. 106, 094312 (2009).

18. S. A. Angayarkanni and J. Philip, Adva. Colloid Inter. Sci. 225, 146 (2015).

19. M. Gnaneswara Reddy and O. D. Makinde, J. Mole. Liq. 223, 1242 (2016).

20. N. S. Akbar, M. Raza, and R. Ellahi, J. Appl. Fluid Mech. 9, 1721 (2016).

21. K. P. Tikhomolova, Drying Technol. 12, 1243 (1993).

22. Kh. S. Mekheimer and Y. A. Elmaboud, Phys. Lett. A 372, 1657 (2008).

23. Kh. S. Mekheimer and Y. A. Elmaboud, Physica A 387, 2403 (2008).

24. M. H. Haroun, Comput. Mater Sci. 39, 324 (2007). 
25. T. Hayat and N. Ali, Math. Comput. Model 48, 721 (2008).

26. W. A. Khan and I. Pop, Int. J. Heat Mass Trans. 53, 2477 (2010).

27. H. Ginsburg, J. Theo. Biol. 37, 389 (1972).

28. V. Sansalone, J. Kaiser, S. Naili, and T. Lemaire, Biomech. Model. Mechan. 12, 533 (2013).

29. E. A. Marshall, J. Theo. Biol. 66, 107 (1977).
30. A. Mondal and G. C. Shit, Euro. J. Mech. B/Fluids 60, 1 (2016).

31. Saeid Hadian, Saeid Movahed, and Nader Mokhtarian, World Appl. Sci. J. 17, 666 (2012).

32. A. Garai and S. Chakraborty, Int. J. Heat Mass Trans. 52, 2660 (2009).

33. G. C. Shit, A. Mondal, A. Sinha, and P. K. Kundu, Colloids and Surfaces A: Phys. Eng. Asp. 506, 535 (2016). 